\title{
Prévalence des mariages précoces dans les régions septentrionales du Mali.
}

\section{Prévalence of the early marriages in the septentrional areas of Mali.}

AG IKNANE A 1, CISSE I 2, TOURE M 3, KANTE S 4, DIAWARA A 5, THIERO T H 6, SOW A 7

1: Maître assistant en santé publique, à la FMPOS, INRSP Bamako

2. Sociologue, chercheur à l'IER, Bamako - Mali

3. Maître assistant en Gyneco-obstétrique à la FMPOS, médecin chef CSRéf Commune IV, Bamako

4. Médecin généraliste, Bamako - Mali

5. Maître assistant en santé publique à la FMPOS, Directeur de l'ANEH, Bamako

6. Biologiste à l'INRSP, Chef de cabinet MESSRS, Bamako

7. Professeur de gyneco-obstétrique à la FMPOS, Bamako

\section{Résumé}

Si la pratique des mariages précoces a reculé au niveau mondial au cours des 30 dernières années, elle est encore répandue chez pays les plus pauvres et en zones rurales. C'est en Asie du Sud, en Afrique de l'ouest et en Afrique centrale qu'elle est la plus fréquente.

Une étude prospective de type descriptive, ayant utilisé une approche quantitative et qualitative a porté sur la pratique des mariages précoces d'octobre 2007 à novembre 2009 dans les régions de Mopti, Gao et Tombouctou. La pratique du mariage précoce est restée assez importante dans les trois régions étudiées avec des niveaux de prévalence de $52,3 \%$ à Mopti, $63,3 \%$ à Gao et $58 \%$ à Tombouctou. La pratique est resté fortement endogamique avec $85 \%$ de mariage précoce pratiqués au sein de la même famille à Tombouctou, $71,6 \%$ à Mopti, et $37,2 \%$ à Gao. Toutes les ethnies étaient concernées. La principale raison du mariage précoce était la peur des grossesses illégitimes avec $51,7 \%$ à Tombouctou, $42,8 \%$ à Gao et $41,1 \%$ à Mopti Mots clés : Mariages précoces, Mopti, Gao, Tombouctou

\section{Summary}

If the practice of the early marriages moved back on a world level during 30 last years, it is still widespread at the poorest countries and in rural zones. It is in South Asia, West Africa and central Africa which it is most frequent.

An exploratory study of descriptive type, having used a quantitative and qualitative approach related to the practice of the early marriages from October 2007 at November 2009 in the areas of Mopti, Gao and Tombouctou. The practice of the early marriage remained rather significant in the three areas studied with levels of prévalence of $52,3 \%$ to Mopti, $63,3 \%$ with Gao and $58 \%$ with Tombouctou. The practice remained strongly endogamic with $85 \%$ de early marriage practised $\mathrm{S}$ within the same family with Tombouctou, $71,6 \%$ with Mopti, and $37,2 \%$ with Gao. All the ethnos groups were concern ée $\mathrm{S}$. The principal reason of the early marriage was the fear of the illegitimate pregnancies with $51,7 \%$ with Tombouctou, $42,8 \%$ with Gao et $41,1 \%$ in Mopti

Key words: Early Marriages, Mopti, Gao, Tombouctou

Introduction :

Tout mariage qui a été conclu et consommé à un âge inférieur à 18 ans pour la fille qui n'est pas encore arrivée à la maturation complète de ses organes est dit précoce [1]. Lorsqu'il a été conclu avant l'âge de 15 ans, il aura été très précoce [1]. Le mariage devrait relever d'un choix. Ce principe juridique fut reconnu depuis l'époque romaine et a été consacré par les instruments internationaux des droits humains [2,1,3]. Malgré tout, de nombreuses filles se marient encore sans possibilité d'exercer leur droit de choisir . [4,5, 6,1,7]. Le mariage précoce revêt de nombreuses formes et relève de divers facteurs [8]. II constitue une violation des droits humains aussi bien pour les filles que les garçons. Cette violation dont les effets sur le plan physique, intellectuel, psychologique, émotionnel, met fin aux possibilités d'éducation et de croissance individuelle de l'enfant $[8,9,10,11,12]$.

En Europe occidentale et Amérique du nord le mariage a lieu tardivement. L'âge moyen du mariage pour les femmes d'Europe du 16 è siècle est de 24 ans et de 27 ans au 18è siècle [14]. Au Moyen-Orient et en Afrique du Nord, le mariage précoce est moins commun qu'en Asie du Sud ou en Afrique sub-saharienne. Malgré le peu de données disponibles, nous savons que dans les Emirats arabes unis $55 \%$ des femmes de 20 ans sont mariées, et ce chiffre est de $42 \%$ au Soudan [5].

Le mariage précoce est généralement plus répandu en Afrique Occidentale et Centrale avec respectivement $40 \%$ et $49 \%$ comparé à Afrique Orientale avec $27 \%$ et l'Afrique du Nord et du Sud avec 20\% [1,13]. Un grand nombre de ces jeunes mariées entrent comme $2^{\mathrm{e}}$ ou $3^{\mathrm{e}}$ épouse dans des ménages polygames. [13]. II existe peu de statistique sur l'âge du mariage entre 10 et 14 ans en dehors du Bengladesh ave une prévalence de $5 \%$ entre 10 et 14 ans $[13,12]$. La coutume de marier les filles de "bonne heure" est très répandue en Afrique subsaharienne et en Asie du Sud mais aussi dans certaines parties d'Amérique latine et certaines zones d'Europe orientale.

Au Mali bien que la pratique semble ancestrale, aucune étude de prévalence n'a encore été réalisée. C'est dans ce contexte que la présente étude a été réalisée pour évaluer la fréquence des mariages précoces dans les régions septentrionales $\mathrm{du} \quad$ Mali (Mopti,Tombouctou,Gao).

Matériels et méthode :

Une étude transversale descriptive s'est déroulée du 24 octobre au 12 novembre 2007 pour les régions de Mopti et Gao et du 20 juillet au 02 août 2008 pour la région de 
Tombouctou. Une revue documentaire a été réalisée pour la collecte des données quantitatives dans les centres de santé de cercle et les centres de santé communautaires. Les données qualitatives ont été collectées au moyen d'entretiens individuels et de focus groupe auprès des femmes et filles de 9 ans et plus, des leaders communautaires et communaux et des associations et institutions juridiques des localités étudiées.la collecte de données quantitatives ont été réalisées au niveau des différentes formations sanitaires pour évaluer les conséquences des mariages précoces sur la santé de la reproduction et déterminer le coût de la prise en charge de ses conséquences en particulier pour les fistules vésico-vaginales.

$\mathrm{Au}$ plan éthique, le consentement des leaders communautaires et des différents informateurs clé a été requise avant la collecte des données. Une restitution des résultats de l'étude a été réalisée au niveau de chacun des villages ayant fait l'objet de l'étude.

\section{Résultats}

Notre étude a concerné un échantillon de 1017 femmes et filles de 9 ans et plus dont 291 à Mopti, 388 à Gao et 338 à Tombouctou (Tableau I). Elle a concerné 3 régions, 7 districts sanitaires et 12 aires de santé (tableau I). Le niveau d'instruction était très faible avec un niveau d'analphabétisme de $90,2 \%$ à Tombouctou, $85 \%$ a Mopti et $70,7 \%$ a Gao. Près de $25 \%$ seulement de femmes de Gao avaient atteint le niveau du premier cycle de l'enseignement fondamental contre 9,8 \% seulement à Mopti et 9,4\% à Tombouctou (Tableau IV). La fréquence des mariages précoces était élevée dans le septentrion malien avec $52,3 \%$ dans les régions de Mopti, $58 \%$ à Tombouctou et $63,3 \%$ à Gao. La pratique du mariage précoce a été observée dans toutes les ethnies des trois régions étudiées (Tableau II). A à Mopti, le mariage a été précoce chez $31 \%$ de peulh et $30 \%$ de Dogon, $47 \%$ de tamasheks et $42 \%$ de sonrhaï à Gao et $37 \%$ d'arables et $18,6 \mathrm{M}$ de sonrhaï à Tombouctou (tableau II). Le mariage a été très précoce dans la région de Gao avec 19,4\%, suivi de Tombouctou avec 14,8\% et Mopti avec 12,9\% (Tableau III).

La principale raison du mariage précoce était la peur des grossesses illégitimes avec $51,7 \%$ à Tombouctou, 42,8 $\%$ à Gao et $41,1 \%$ à Mopti. La pratique de l'endogamie a été retrouvée au niveau des mariages précoces réalisés. La pratique se faisait majoritairement en fonction du lien de parenté avec $85 \%$ de mariage précoce réalisés au sein de la même famille à Tombouctou, $71,6 \%$ à Mopti, et $37,2 \%$ à Gao

\section{Commentaires et discussion}

La prévalence des mariages précoces était assez élevée dans les régions septentrionales du Mali avec 63,3\% à Gao, $58 \%$ à Tombouctou et $52,3 \%$ à Mopti. Nos résultats sont plus élevés que ceux observé au Guatemala de $8 \%$ chez les garçons contre $24 \%$ chez les filles [5]. II en est de même que ceux de l'étude réalisée en mars 2001 par le Fonds des Nations Unies pour la Population [5 ] qui donne pour l'ensemble du pays une prévalence des mariages précoces de $5 \%$ chez les garçons contre $50 \%$ chez les filles. Nos résultats, sont également plus élevés que ceux observés dans certaines parties d'Europe centrale et orientale, en particulier chez les Romains [14] et en Macédoine où $27 \%$ des femmes mariées au cours de l'année 1994 avaient entre 15 et 19 ans [15].

Notre étude a montré que le mariage a été très précoce (avant l'âge de 15 ans) dans $13 \%$ des cas à Mopti, $15 \%$ à Tombouctou et $19 \%$ à Gao. Nos résultats sont plus élevés que ceux observés en Amérique du Nord, en Europe et en Océanie. Dans les pays industrialisés, (Amérique du Nord, Europe et Océanie), peu de femmes se marient avant 18 ans, seulement $4 \%$ aux Etats-Unis et $1 \%$ en Allemagne [6].

Nos résultats par contre sont moins élevés que ceux observés dans la plupart des pays asiatiques, d'Afrique centrale et de l'Ouest. En Asie, les structures matrimoniales sont bien plus différenciées. L'Afghanistan et le Bangladesh, où respectivement $54 \%$ et $51 \%$ des filles sont mariées avant 18 ans représentent des cas extrêmes [7]. Au Népal, où l'âge moyen du premier mariage est 19 an, 28,7\% des filles sont mariées avant 10 ans et $40 \%$ avant 15 ans [15]. En Chine la proportion des mariages précoces a diminué de $35 \%$ durant les années 70 pour passer ensuite de $13 \%$ en 1979 à $18 \%$ en 1987[7,11].

Une étude récente de I'UNICEF menée dans six pays d'Afrique de l'Ouest [6] révèle que $44 \%$ des femmes nigériennes se sont mariées avant l'âge de 15 ans. Au Bangladesh, on compte 5 garçons mariés entre 15 et 19 ans pour 51 filles du même âge en 2001 d'après la même étude [7]. Ces résultats sont plus élevés que ceux retrouvés par notre étude. Le mariage précoce est généralement plus répandu en Afrique centrale et occidentale, concernant respectivement $40 \%$ et $49 \%$ des filles de moins de 19 ans, par rapport à $27 \%$ en Afrique orientale et $20 \%$ en Afrique du Nord et du Sud [11.]

\section{CONCLUSION}

Le mariage précoce est resté une pratique assez repandue dans les régions septentrionales du Mali ,avec des niveaux d eprévalence de plus de $50 \%$. II représente une grave menace pour le bien-être des enfants. Si cette pratique concerne filles et garçons, ce sont toutefois les droits les plus fondamentaux de l'épouse enfant en matière de survie et de développement qui sont méconnus.

L'abandon de la pratique du mariage précoce passe nécessairement par des actions ciblées en direction des groupes sociaux concernés allant dans le sens d'une communication efficace et dynamique pour le changement de comportement des parents afin qu'ils prennent conscience des conséquences réelles que peut induire cette pratique, une plus grande implication des autorités communales et des hommes dans la prévention et la lutte contre la pratique; L'implication des médias et des communicateurs traditionnels pour assurer une plus large diffusion des messages de plaidoyer en faveur de l'adoption du nouveau code de mariage et de la tutelle et de l'abandon de la pratique des mariages précoces. 
La recherche de l'équilibre entre la pression sociale familiale et le souci d'émancipation sociale de la fille à travers la sensibilisation des parents et des leaders communautaires, la promotion d'activités génératrices de revenus et ou d'allègement des tâches domestiques pour les mères,

\section{Bibliographie}

1. UNICEF. Le mariage précoce, les points essentiels, Digest Innocenti, $n^{\circ} 7$, mars 2001

2. World Bank. Mariage Patterns, Wallchart, Département des Affaires Economiques et Sociales de l'ONU, 2000.

3. Mensch, Barbara S, Judith Bruce, Margaret S, Greene. "The Uncharted Passage: Girls Adolescence in the Developing World, Conseil de la population, New York, $71 \mathrm{p}$.

4. Kabir R. "Adolescent Girls Bangladesh", UNICEF Dhakah, 1998.

5. Westoff, Charles F. "Age at Marriage, Age at First Birth, and Fertility in Africa", World Bank, Technical paper n 169, Banque Mondiale, Washington DC, 1992.

6. Assani Aliou, Etude sur les mariages précoces et grossesses précoces au Burkina-Faso, Cameroun, Gambie, Liberia, Niger et Tchad, UNICEF WCARO, Abidjan, 2000.

7. Indralal W. "The Ireland of Asia, Trends in Marriage Timing in Sri Lanka", Asia-Pacific Population Journal, (1997), ONU 1991, vol . 12, n² 2.

Tableau III : Répartition de la population étudiée selon l'age

\begin{tabular}{|l|c|c|c|}
\hline \hline $\begin{array}{l}\text { Les tranches } \\
\text { d'age\% }\end{array}$ & Mopti & Gao & Tombouctou \\
\hline 9-14 ans & 12,9 & 19,4 & 14,8 \\
\hline 15-17 ans & 12,9 & 12,3 & 10,9 \\
\hline 18 et plus & 74,2 & 68,3 & 74,3 \\
\hline Total & $\mathbf{5 2 , 3}$ & $\mathbf{6 3 , 3}$ & $\mathbf{5 8}$ \\
\hline
\end{tabular}

8. CIAF. Le Mariage précoce, Bulletin $n^{\circ} 15$, Décembre 1993.

9. Barton, Tom, Alfred Mutiti.. "Northern Uganda Psychosocial Needs Assessment Report", République d'Ouganda et UNICEF; "AIDS Orphans" (2000) Information sheet on the HIVIAIDS emergency, UNICEF ESARO, Nairobi (1998).

10. Amin. Kishor S, Johnson K. "Profiling Domestic Violence: A Multi - Country Study". Calverton, MD: ORC Macro, 2004

11. Unicef, Mariage précoce: conjointe, enfant, document électrique, www.unicef_icdc.org, consulté le 24/01/09.

12. Berhane RAS-WORK. Le Mariage précoce, IAC * $\mathrm{Cl}-\mathrm{AF}$, Communication présentée à la Gème Conférence de l'Organisation Internationale de la Francophonie à Caraquet, Nouveau-Brunswick, Canada.

13. Westoff, Charles F. "Age at Marriage, Age at First Birth, and Fertility in Africa', World Bank Technical Paper n 169, (1992).

14. UNICEF. Rapport sur la situation des enfants dans le monde, novembre 2006.

15. Jones Gavin. "Population Dynamics and Their Impact on Adolescents in the ESCAP Region, in Asia-Pacific» Population Journal, 1997, vol. 12, nº 3.

Tableau IV : Répartition de la population étudiée selon le niveau d'instruction \%

\begin{tabular}{|l|c|c|c|}
\hline Niveau d'instruction & Mopti & Gao & Tombouctou \\
\hline Non alphabétisées $\%$ & 85 & 70,7 & 90,2 \\
\hline Niveau 1er cycle $\%$ & 9,8 & 24,7 & 9,5 \\
\hline Niveau 2er cycle & 2,4 & 4,6 & 0,3 \\
\hline Autres & 2,8 & 0 & 0 \\
\hline Total & 100 & 100 & 100 \\
\hline
\end{tabular}


Tableau I : Entretiens réalisés auprès des femmes ou filles de 9 ans et plus

\begin{tabular}{|c|c|c|c|}
\hline Région & Cercle/CSRéf & Aire de santé & $\begin{array}{l}\text { Femmes ou filles } \\
\text { De } 9 \text { ans et plus }\end{array}$ \\
\hline \multirow[t]{4}{*}{ MOPTI (291) } & Mopti & Fatoma & 61 \\
\hline & & Sofroulaye & 55 \\
\hline & Bankass & Doundé & 91 \\
\hline & & Dimbal & 84 \\
\hline \multirow[t]{3}{*}{ GAO ( 388) } & Gao & Haoussa Foulane & 83 \\
\hline & & Forgho & 125 \\
\hline & Ansongo & Tin Hamma & 180 \\
\hline \multirow{5}{*}{$\begin{array}{l}\text { Tombouctou } \\
(338)\end{array}$} & Diré & Kondi & 55 \\
\hline & & Gari & 57 \\
\hline & Rharous & Gourma Rharous & 25 \\
\hline & & Haribomo & 100 \\
\hline & Niafunké & Fittouga & 101 \\
\hline Total & 7 & 12 & 1017 \\
\hline
\end{tabular}

Tableau II : Répartition de la population étudiée selon l'ethnie en \%

\begin{tabular}{lrrr}
\hline & Gao & Mopti & Tombouctou \\
$\mathrm{n}=291$ & $\mathrm{n}=388$ & $\mathrm{n}=338$ \\
\hline Tamashek & 47 & 0 & 14,5 \\
Sonrai & 42 & 0 & 18,6 \\
Peuhl & 11 & 31 & 17,5 \\
Arabe & 0 & 0 & $\mathbf{3 7 , 0}$ \\
Bambara & 0 & 10 & 8,6 \\
Dogon & 0 & 30 & 0 \\
Bozo & 0 & 20,6 & 0 \\
Marka & 0 & 8,4 & 0 \\
Autres & 0 & 0 & 3,8 \\
Total & 100,0 & 100,0 & 100,0 \\
\hline
\end{tabular}

